\title{
Free gold clusters: beyond the static, monostructure description
}

\author{
Elizabeth C. Beret, Luca M. Ghiringhelli and Matthias Scheffler*
}

\author{
Received 25th February 2011, Accepted 24th March 2011 \\ DOI: $10.1039 / \mathrm{clfd00027f}$
}

\begin{abstract}
The thermodynamical stability of free, pristine gold clusters at finite temperature, and of cluster+ligands complexes at finite temperature and in the presence of an atmosphere composed of $\mathrm{O}_{2}$ and $\mathrm{CO}$, is studied employing parallel tempering and $a b$ initio atomistic thermodynamics. We focus on $\mathrm{Au}_{13}$, which displays a significant fluxional behavior: Even at low temperature $(100 \mathrm{~K})$ this cluster exhibits a multitude of structures that dynamically transform into each other. At finite temperature, the preference of this cluster for three-dimensional versus planar structures is found to result from entropic effects. For gold clusters containing one to four gold atoms in an $\mathrm{O}_{2}+\mathrm{CO}$ atmosphere, we apply ab initio atomistic thermodynamics. On the basis of these considerations, we single out a likely reaction path for $\mathrm{CO}$ oxidation catalyzed by gold clusters.
\end{abstract}

\section{Introduction}

The discovery that nanosized gold particles are good catalysts for carbon monoxide oxidation at rather low temperatures, ${ }^{1,2}$ as well as for other oxidation reactions, ${ }^{3-7}$ has been attracting the interest and excitement of a large number of researchers in the scientific community for more than twenty years. ${ }^{8-23}$

Carbon monoxide oxidation, $\mathrm{CO}+\frac{1}{2} \mathrm{O}_{2} \rightarrow \mathrm{CO}_{2}$, does not happen spontaneously in the gas phase because it breaks the spin conservation rule $\left(\mathrm{O}_{2}\right.$ has a triplet ground state, while both $\mathrm{CO}$ and $\mathrm{CO}_{2}$ are singlets). In recent years, several structures at various cluster sizes and stoichiometries have been studied by means of density-functional theory (DFT). ${ }^{1,10,13,22}$ Such studies share a common strategy: They aim at finding energetically ordered lists of isomers at the chosen compositions, and the lowest energy structures are often used as starting points for discussing a catalytic process mediated by the cluster. However, as we will stress below, a static, monostructure description of nanosized particles is likely to miss some important properties and processes. Furthermore, knowing the energetic hierarchy of the cluster+ligands complexes at a given stoichiometry is not sufficient to understand the system at the realistic conditions where catalysis occurs. In heterogeneous catalysis, the catalyst is exposed to an atmosphere of the gas-phase reactants at finite temperature and pressure. The present contribution shows the necessity of accounting for both temperature and pressure effects, even to determine the structure of the catalyst. This is a preliminary but necessary step before taking chemical reaction kinetics into consideration.

To this purpose, we proceed in steps. We first show that the apparently simple question "what is the structure of the pristine gold cluster of size $N$ ?" requires an answer that involves ensemble averages at the temperature of interest. As an example, we present the case of $\mathrm{Au}_{13}$, which exhibits a significant fluxional behavior. The term fluxional reflects that the potential-energy surface (PES) of the cluster has 
several minima differing by just a few $\mathrm{meV}$, and that these minima are separated/by Online small barriers. When the height of the barriers is comparable with the average kinetic energy of the cluster at a given temperature, the cluster visits many minima in short timescales, say a few ps. At increasing temperatures, such clusters will exhibit first an ensemble of different rigid structures whose population is dictated by their Boltzmann weight, then fluxional behavior, and then the liquid state, where a continuous rearrangement of bonds takes place.

Furthermore, we show how the presence of a single ligand $\left(\mathrm{Au}_{13}+\mathrm{CO} \rightarrow\right.$ $\left.\mathrm{Au}_{13} \mathrm{CO}\right)$ subverts the energetic hierarchy of $\mathrm{Au}_{13}$ geometries leading to a very different statistical ensemble.

In a third step, we also consider finite temperature and finite pressure of the reactants in the gas phase. A theoretical approach for the prediction of the thermodynamically stable and metastable catalyst+ligand structures has been developed initially for bulk semiconductors ${ }^{24,25}$ and more recently for surface oxides. ${ }^{26,27}$ Here we extend this method, called ab initio atomistic thermodynamics, to gas-phase clusters in an environment of $\mathrm{O}_{2}$ and $\mathrm{CO}$. The candidate structures are extensively sampled by means of the basin hopping technique. ${ }^{28}$ This is rather efficient for not too big system sizes. In the original method conceived for bulks and surfaces, the temperature dependence was considered only in the partition function of the reactant gas; in other words, the free energy of the catalyst was approximated in terms of its electronic energy. While this is typically a good assumption for bulk materials ${ }^{26}$ the free energy of gas-phase clusters and cluster+ligands complexes depends significantly on temperature.

$A b$ initio atomistic thermodynamics yields phase diagrams, which identify structures that are probably relevant for catalysis at the realistic temperature and pressure of interest. We further extend the method by considering the possibility of $\mathrm{CO}_{2}$ desorption. On the basis of this thermodynamic analysis, we can single out likely reaction mechanisms.

In many experimental studies, gold clusters are deposited on an oxide surface, and the nature of the support has a noticeable influence on the catalytic activity (see for instance Ref. 17). Other experiments aim at a "clean room" analysis of the intrinsic cluster properties and are conducted for clusters in the gas phase. Due to instrumental constraints, experiments with free clusters usually dealt with ionized species, and whether the cluster bore a positive or a negative electric charge also affected its catalytic properties (see Ref. 18 and references therein). Recently, also experimental studies of neutral clusters became possible. ${ }^{29}$ In this contribution we study free, neutral gold clusters by DFT-based thermodynamics and statistical mechanics, in order to isolate their intrinsic catalytic properties from the influence of a support.

\section{Calculation methods}

For various compositions containing different numbers of $\mathrm{Au}, \mathrm{CO}$, and $\mathrm{O}$, we sampled their structures using basin hopping ${ }^{28}$ and parallel tempering ${ }^{30,31}$ on the basis of DFT total energies and forces. We employed the FHI-aims code ${ }^{32}$ using the PBE exchange-correlation functional. ${ }^{33,34}$ Scalar-relativistic corrections have been applied. ${ }^{32,35}$ Dispersion interactions, which play a noticeable role in describing the structure of gold clusters, ${ }^{36}$ as well as their interaction with $\mathrm{CO}$ ligands, ${ }^{37}$ are included in our calculations by means of a $C_{6}[n] / r^{6}$ tail correction to the PBE energy, where the $C_{6}[n]$ coefficients are derived from the self-consistent electron density $n .^{38}$ We will refer to this functional as PBE+vdW.

We have studied $\mathrm{Au}_{N} \mathrm{O}_{x}(\mathrm{CO})_{y}$ clusters containing between one and four gold atoms, and up to eight $\mathrm{O}$ atoms and/or four $\mathrm{CO}$ molecules. The structures of these clusters were sampled by means of basin hopping. In their ground electronic states, gas-phase $\mathrm{Au}_{N}$ clusters are doublets (clusters with odd $\mathrm{N}$ ) and singlets (even $\mathrm{N}$ ), $\mathrm{CO}$ is a singlet, and $\mathrm{O}, \mathrm{O}_{2}$ are triplets. Thus, the cluster+ligands structures can have different spin states, and we have sampled several possibilities $(2 S+1=2,4,6$ 
for clusters with odd number of total electrons, i.e. odd $N$, and 1, 3, 5 for clusters Online with even $\mathrm{N}$ ). The stabilities of the structures produced in this sampling are obtained as a function of the environmental conditions (temperature and partial pressures of the reactive gases) by means of ab initio atomistic thermodynamics, which will be described in the following.

We consider that the $\mathrm{Au}_{N}$ clusters reach thermal equilibrium with the $\mathrm{O}_{2}$ and $\mathrm{CO}$ gases, and $\mathrm{Au}_{N} \mathrm{O}_{x}(\mathrm{CO})_{y}$ structures are formed. However, $\mathrm{CO}$ and $\mathrm{O}_{2}$ are not in equilibrium with each other. Such a constrained thermodynamic equilibrium neglects the non-catalytic $\mathrm{CO}+\frac{1}{2} \mathrm{O}_{2} \rightarrow \mathrm{CO}_{2}$ reaction, which is forbidden by a spin selection rule.

For each set of environmental conditions $\left(T, p_{\mathrm{O}_{2}}, p_{\mathrm{CO}}\right)$ the most stable $\mathrm{Au}_{N} \mathrm{O}_{x}(\mathrm{CO})_{y}$ compositions are those with the minimum free energy of formation:

$$
\Delta G_{f}\left(T, p_{\mathrm{O}_{2}}, p_{\mathrm{CO}}\right)=G_{\mathrm{Au}_{N} \mathrm{O}_{x}(\mathrm{CO})_{y}}(T)-G_{\mathrm{Au}_{N}}(T)-x \mu_{\mathrm{O}}\left(T, p_{\mathrm{O}_{2}}\right)-y \mu_{\mathrm{CO}}\left(T, p_{\mathrm{CO}}\right)
$$

$G_{\mathrm{Au}_{N} \mathrm{O}_{x}(\mathrm{CO})_{y}}$ and $G_{\mathrm{Au}_{N}}$ are the free energies of the cluster+ligands and of the pristine cluster, and $\mu_{O}, \mu_{C O}$ are the chemical potentials of oxygen and carbon monoxide. The chemical potential of oxygen is $\mu_{O}=\frac{1}{2} \mu_{O_{2}}\left(T, p_{\mathrm{O}_{2}}\right)$. The free energies of the cluster+ligands and of the pristine cluster are obtained from their partition functions ${ }^{39,40}$ including translational, rotational, vibrational, electronic, and configurational degrees of freedom. In the case of surface oxides, the change in the translational, rotational, and vibrational components due to ligand adsorption is typically negligible, and it can be disregarded in eqn (1). This is not the case of metal clusters in the gas phase, for which these contributions can change significantly upon ligand adsorption. The chemical potentials of $\mathrm{CO}$ and $\mathrm{O}_{2}$ are also obtained from their partitions functions. Using eqn (1) we find out which $\mathrm{Au}_{N} \mathrm{O}_{x}(\mathrm{CO})_{y}$ compositions are the most stable ones at given environmental conditions $\left(T, p_{\mathrm{O}_{2}}, p_{\mathrm{CO}}\right)$. The results can be plotted as phase diagrams.

For many $\mathrm{Au}_{N} \mathrm{O}_{x}(\mathrm{CO})_{y}$ compositions considered in the sampling we find that the lowest-energy structure at $0 \mathrm{~K}$ is closely followed by several other isomers within energies just a few tens of meV higher. This implies that at finite temperature the $\mathrm{Au}_{N} \mathrm{O}_{x}(\mathrm{CO})_{y}$ cluster cannot be properly described by only a single structure, but rather consists on a distribution of isomers. The population of a certain conformer of free energy $G_{i}$ with respect to the structure with the lowest free energy $G_{0}$ at a given temperature $T$ is given by the Boltzmann factor

$$
B_{i}(T)=\exp \left(-\frac{G_{i}-G_{0}}{k_{B} T}\right)
$$

For each cluster composition we consider those isomers with a relative population of at least $5 \%\left(B_{i}>0.05\right)$.

Parallel tempering was used in conjunction with Born-Oppenheimer molecular dynamics (BO-MD) to study the structures of pristine clusters and cluster+ligands at larger sizes. In this contribution, we discuss results from parallel tempering only for clusters containing thirteen gold atoms. The time step for the BO-MD runs was $10 \mathrm{fs}$ for the pristine $\mathrm{Au}_{13}$ cluster and 2 fs for the $\mathrm{Au}_{13} \mathrm{CO}$ complex. The shorter time step for the cluster $+\mathrm{CO}$ system is dictated by the $\mathrm{C}-\mathrm{O}$ bond stretching frequency, that is much higher than the highest vibrational frequencies in pristine gold clusters. The temperature of the simulations was controlled by means of the Bussi-Donadio-Parrinello thermostat. ${ }^{41}$ We have used 16 replicas with temperatures ranging from $100 \mathrm{~K}$ to $1000 \mathrm{~K}$, and the swap between configurations was attempted every five MD steps. In comparison to a low frequency of attempt swapping, such a high attempt frequency has been shown to yield a better efficiency of phase-space sampling. ${ }^{42}$ Further details about the parallel tempering simulations and their analysis will be published elsewhere. The use of parallel tempering for sampling clusters' 
phase space is useful for two reasons. By selecting structures that are visited at Vodw Online temperature and geometrically optimizing them, one gathers a large set of isomers in a fully unbiased manner. Furthermore, the sampling at each temperature is by construction canonical, hence the average value of any generalized coordinate is an estimator of the canonical expectation value of that variable.

We have assessed the accuracy of our PBE+vdW approach by comparing results for certain clusters with those of $\operatorname{CCSD}(T)$ calculations. The details of these calculations will be discussed elsewhere. ${ }^{43}$ The error in the binding energy of $\mathrm{Au}_{2}$ at $\mathrm{PBE}+\mathrm{vdW}$ with respect to the $\operatorname{CCSD}(\mathrm{T})$ value is small $(-0.06 \mathrm{eV})$. There is a significant and well-known error of DFT-GGA when describing the total energy of an $\mathrm{O}$ atom or $\mathrm{O}_{2}$ molecule, and we find a similar error in the total energies of the $\mathrm{Au}_{N} \mathrm{O}_{x}$ clusters. Since we deal with energy differences, $\Delta E_{f}=E\left(\mathrm{Au}_{N} \mathrm{O}_{x}\right)-E\left(\mathrm{Au}_{N}\right)-\frac{x}{2} E\left(\mathrm{O}_{2}\right)$, both errors are partially compensated. The $\mathrm{PBE}+\mathrm{vdW}$ binding energies of gold clusters to $\mathrm{O} / \mathrm{O}_{2}$ ligands are about $0.45 \pm$ $0.1 \mathrm{eV}$ too strong, in comparison to $\operatorname{CCSD}(\mathrm{T})$ energies. Since the formation energies of all the clusters containing oxygen are subjected to an overestimation of $0.45 \mathrm{eV}$, the conclusions derived from comparison of different clusters will not be affected by this error. There is only an uncertainty of $\pm 0.1 \mathrm{eV}$ in the PBE $+\mathrm{vdW}$ formation energies. As a consequence, the regions in the phase diagrams might actually be wider or thinner than predicted from our calculations, and very thin regions might even disappear.

In a separate contribution ${ }^{44}$ we have computed the vibrational spectra of free gold clusters at finite temperature using this same PBE+vdW calculation level. The comparison of these theoretical spectra with experimental ones is more than satisfying, which constitutes a proof that gold clusters are well described at the PBE $+\mathrm{vdW}$ level.

\section{Fluxional clusters}

Identifying the lowest-energy isomer for $\mathrm{Au}_{13}$ has been the subject of several publications in the past years. ${ }^{4-50}$ The reason of the curiosity raised by this particular size is that the most symmetric structures with 13 particles, the cuboctahedron and the icosahedron, are not at all the most stable isomers: Their total energies are more than $1 \mathrm{eV}$ higher than the most stable structures.$^{50}$ Several low symmetry structures, both threedimensional and planar, have been proposed. At the DFT-GGA level ${ }^{50}$ some planar structures have the lowest energies. In particular for charged gold clusters, the relative stability of planar versus three-dimensional structures has been heavily debated. ${ }^{51,52}$ For these, collision cross-section experiments could be performed. The analysis in Ref. 52 indicates that, albeit DFT-GGA finds as local minima candidate structures that match the experimental measurements, it is poor in describing the relative energetic stability between topologically "very different" structures. In particular, at some of the sizes at which the experiments indicate the presence of a three-dimensional structure, DFT-GGA finds planar structures with lower total energy. On the other hand, possibly more accurate functionals (according to the so-called Jacob's ladder of Perdew) yield a 2D-3D energy hierarchy which is consistent with experiments. For neutral clusters, the discussion in the literature is less rich ${ }^{50}$ since basically no experimental data are available for comparison. Here, we show that the energetic stability of DFT-GGA three-dimensional structures becomes more pronounced by the inclusion of dispersion interactions, and that the free energy, rather than only the total energy, must be taken into consideration for identifying the structures that are present at finite temperature.

\subsection{The importance of dispersion interactions}

In Fig. 1 we show the geometries of all the mechanically stable isomers that we found with a total energy within about $0.1 \mathrm{eV}$ from the most stable structure. The lowestenergy three-dimensional structures included in the red box (isomers $a-c$ ) are 

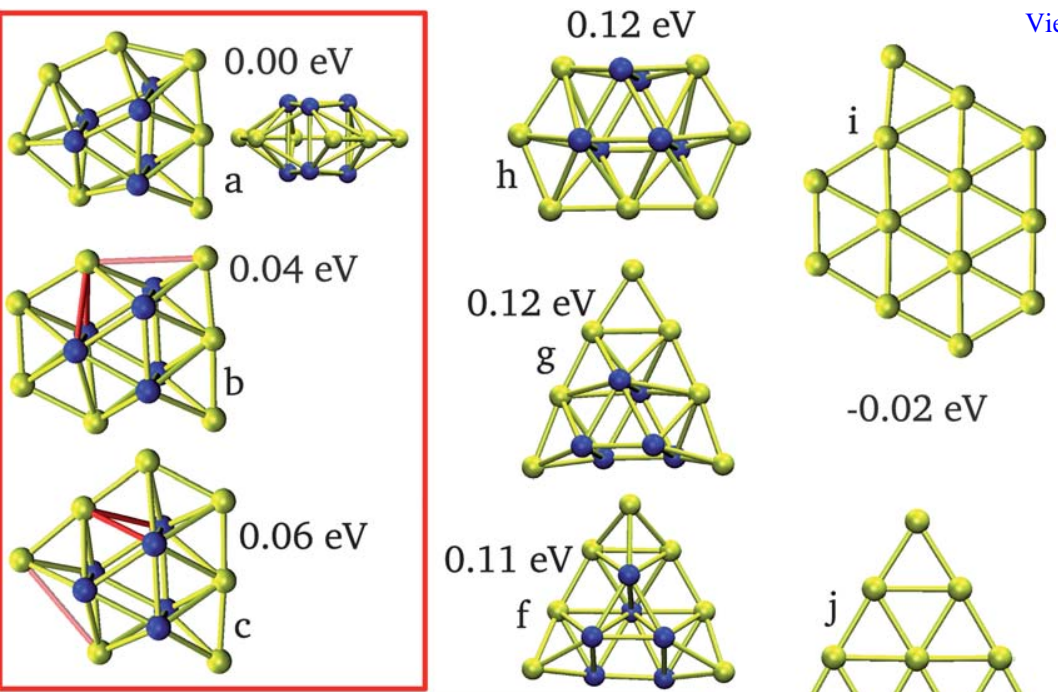

View Online

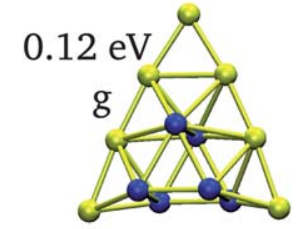

\section{$-0.02 \mathrm{eV}$}

0.1
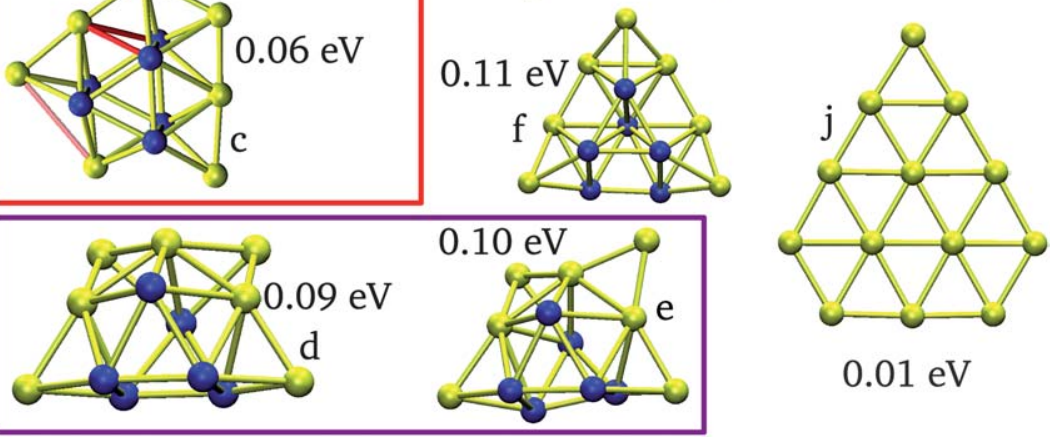

$0.01 \mathrm{eV}$

Fig. 1 Low energy isomers for $\mathrm{Au}_{13}$. The boxes gather members of a set of isomers with low interconversion energy barrier (see text). The numbers indicate the difference in PBE+vdW energy from the lowest-energy three-dimensional isomer. Highlighted in blue are the gold atoms that constitute the triangular prism.

separated by very low barriers. The fact that barriers are low follows from few hundred ps-long constant-temperature Born-Oppenheimer MD runs. Only barriers that are low when compared to the average kinetic energy of the system can be overcome in a few ps, so that structural change can be observed in a MD run at low temperature. These structures can be described as a six-membered triangular prism and an incomplete ring of 7 atoms, all laying in a plane cutting half-way the prism (in the figure we have highlighted in blue the six atoms constituting the prism). This prism motif for $\mathrm{Au}_{13}$ was firstly identified by Gruber et al. ${ }^{50}$ In the figure we mark in red/pink the bonds of isomers $b$ and $c$ that need to break/form in order to transform the structure into the lowest-energy one (i.e. isomer $a$ in Fig. 1). In a MD trajectory at temperature as low as $100 \mathrm{~K}$, these three structures do change into each other. In the purple box there are two different structures (isomers $d$ and $e$ in Fig. 1) yet retaining the prism motif and again separated by a very low barrier, so that they also transform into each other at $100 \mathrm{~K}$. The barriers separating the structure in the red box and those in the purple box are slightly higher; one needs a MD trajectory at $150 \mathrm{~K}$ in order to visit all those five structures (isomers $a-e$ in Fig. 1). To our knowledge, structures like isomers $d$ and $e$ were not found in previous investigations. In a 500 ps long run at $200 \mathrm{~K}$ we also identify other 3D structures (isomers $f-h$ in Fig. 1), which are again based on the prism motif. The parallel tempering sampling finds also two planar structures (isomers $i$ and $j$ in Fig. 1) and their $\mathrm{PBE}+\mathrm{vdW}$ energies are very close to that of the most stable three-dimensional isomer. As it is the case for all planar gold clusters at all sizes, they appear as fragments of a hexagonal planar lattice. 
In order to show the influence of dispersion interactions, we computed the relative Online energies between the most stable planar (isomer $i$ in Fig. 1) and 3D (isomer $a$ in Fig. 1) structures, with both the PBE+vdW functional and the PBE functional. We find that for plain PBE the planar isomer is $0.33 \mathrm{eV}$ more stable than the $3 \mathrm{D}$ one. For PBE+vdW the planar isomer is only $0.02 \mathrm{eV}$ more stable. We also note that, according to $\mathrm{PBE}+\mathrm{vdW}$, the dispersion correction is $2.6 \%$ of the total binding energy for the three-dimensional isomer, while for the planar structure this correction is $1.5 \%$. In general for $\mathrm{Au}_{13}$ all $3 \mathrm{D}$ structures have a dispersion correction that is about $60-70 \%$ larger than for the $2 \mathrm{D}$ isomers.

\subsection{The importance of configurational entropy}

Using the DFT total energy as a stability criterion would be appropriate at $T=0 \mathrm{~K}$. As noted above, even at relatively low temperatures the system visits several conformations, and a complete picture of which isomers are present at a realistic temperature needs the inclusion of configurational entropy, that consists of a conformational part, which reflects the number of occupied energy basins, and a vibrational part, which reflects the shape (width, curvature) of the occupied basins.

Using plain MD trajectories for estimating entropy-related quantities is not efficient at reasonable time scales, due to the fact that leaving a basin is a rare event. In practice, one would mainly sample the vibrational entropy of one or few conformations. With parallel tempering the barriers between basins are overcome at high temperature, and both components of the configurational entropy are efficiently sampled. Using the configurations explored in the parallel tempering runs, we calculated the probability for the system to be either planar or three-dimensional. We found that at $100 \mathrm{~K}$ the probability for a cluster to be planar is about $10 \%$, while at $300 \mathrm{~K}$ the probability drops to $3 \%$.

In summary, $\mathrm{Au}_{13}$, as an example of a rather fluxional cluster, has at low temperature (i.e. around $100 \mathrm{~K}$ ) the structure of a triangular prism with a surrounding planar ring of atoms which continuously rearranges $(a-c$ in Fig. 1). At higher temperatures (i.e. around $150 \mathrm{~K}$ ) also structures with a slightly distorted prism are sampled ( $d$ and $e$ in Fig. 1). At even higher temperatures (more than $200 \mathrm{~K}$ ) other prism+ring structures appear $(f-h$ in Fig. 1$)$. Finally, at $300 \mathrm{~K}$ the cluster is observed to melt into a liquid droplet: The atoms are still covalently bound, but the lifetime of each particular bond is of the order of only one ps. Even as a liquid droplet, $\mathrm{Au}_{13}$ does not have a spherical shape. The instantaneous configuration is oblate (one inertia moment is much smaller than the other two) thus reminding the common feature of all the most stable isomers at $T=0 \mathrm{~K}$. Although some of the planar isomers ( $i$ and $j$ in Fig. 1) have a total energy comparable to that of the lowest-energy three-dimensional structures, they are rarely seen due to entropic reasons. In Fig. 2 we explain this finding in a sketch of the configurational phase space of $\mathrm{Au}_{13}$, where all the $13 \times 3-6=33$ degrees of freedom are collapsed into one coordinate. The planar clusters are in a small region of the phase space, separated by relatively high barriers (the planar clusters melt rapidly in a MD run at $800 \mathrm{~K}$ ) from the region of threedimensional clusters. The basins populated by three-dimensional clusters are shallow, due to the presence of low barriers separating the different minima.

Our conclusions differ from the argument put forward by Koskinen et al. ${ }^{53}$ for anionic gold clusters. These authors suggested that some regions of the phase space (namely, planar clusters) might be difficult to reach when quenching high-temperature clusters: Liquid clusters are three-dimensional and a rapid quenching might lead the system to thermal energies that are too low to overcome the barrier(s) to go to the planar structure. In contrast, our parallel tempering studies imply that an equilibrium argument can be used, that is, the population of planar structures is low due to the topology of the free-energy landscape. Analyzing the free-energy landscape of a gold cluster was already attempted for $\mathrm{Au}_{12}$ by Vargas et $a .^{23}$ by means of metadynamics. ${ }^{54}$ They found nearly equal probability for planar and three-dimensional 


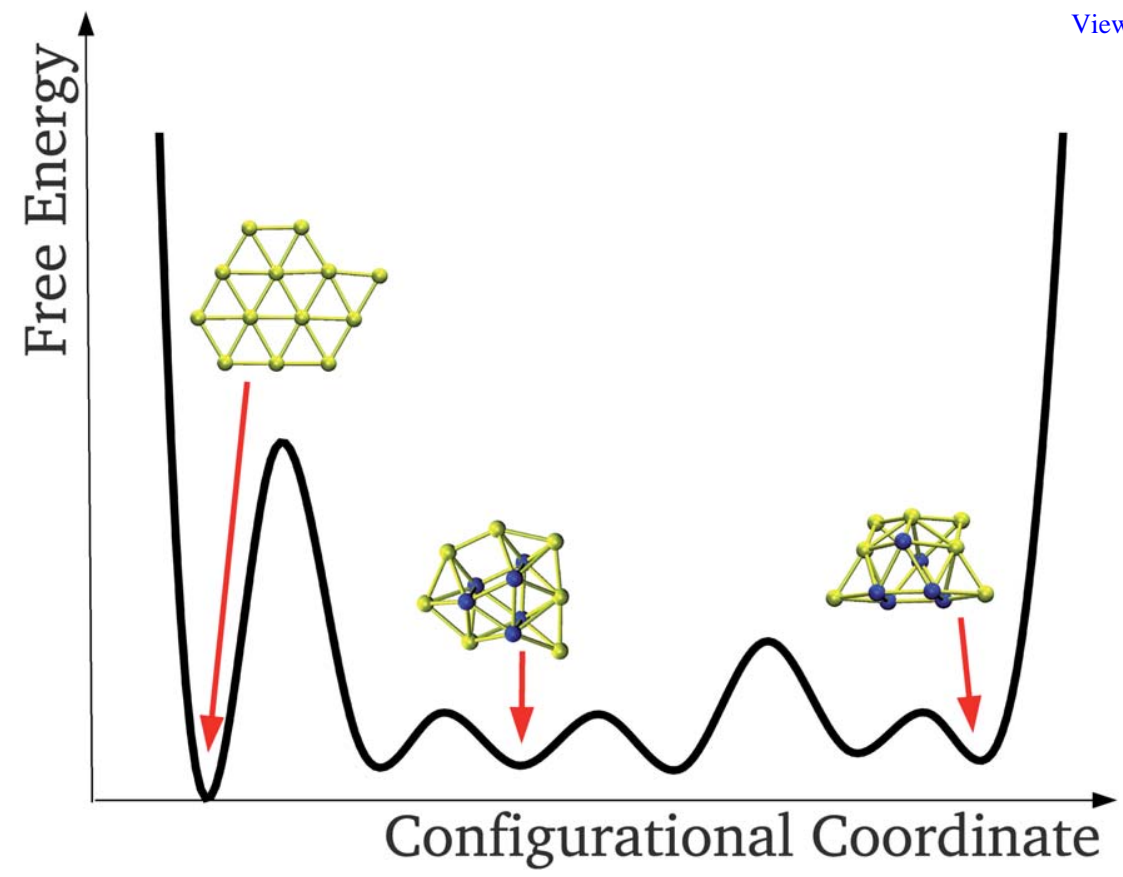

Fig. 2 Schematic view of the free-energy landscape as emerging from our parallel tempering calculations. The planar clusters are confined to a small and stiff basin (left), which is separated by a relatively high energy barrier from the basins containing the most likely three-dimensional structures. The latter structures appear at several shallow minima, separated by barriers that are easily overcome with a modest thermal activation.

structures, whereas the lowest-energy planar structure is $0.2 \mathrm{eV}$ more stable than the lowest-energy three-dimensional one. Here with $\mathrm{Au}_{13}$ we show a neat example where structures with nearly the same total energy have very different statistical weight.

The fluxional character of $\mathrm{Au}_{13}$ is rather extreme within the cluster sizes that we investigated $\left(\mathrm{Au}_{3}-\mathrm{Au}_{20}\right)$ but not at all unique. Clusters as small as $\mathrm{Au}_{3}$ and $\mathrm{Au}_{4}{ }^{44}$ have two low-energy stable isomers, which transform into each other at room temperature. $\mathrm{Au}_{7}^{44}$ presents one stable isomer that nonetheless continuously transforms into an isomer with identical shape and shuffled atoms, and so on.

\section{Ligand adsorption on gold clusters}

\subsection{The influence of ligands on cluster fluxionality}

The fluxional behavior of clusters like $\mathrm{Au}_{13}$ is not restricted to the pristine cluster. Also the configurational phase space of the cluster with ligands is rather rich. As an example, we discuss the configurations of $\mathrm{Au}_{13} \mathrm{CO}$ complexes, as analyzed by means of parallel tempering. The results show that $\mathrm{CO}$ does never dissociate and only one-fold coordinated bonding between the carbon atom and one gold atom occurs in the ensemble. In order to summarize the results, we chose as a descriptor the coordination (actually, the number of Au neighbors) of the gold atom that is bonded to the carbon atom. Table 1 lists the probabilities of finding coordination from two- to five-fold at different temperatures, and in Fig. 3 we show some of these structures. At lower temperature, three- and fourfold coordinated isomers dominate, while at higher temperatures also two-fold coordinated clusters become important, whereas higher-fold coordination becomes 
Table 1 Population of the coordination of the gold atom that is bonded to $\mathrm{CO}$ in $\mathrm{Au}_{13}$ बroew Online

\begin{tabular}{lllll}
\hline$T / \mathrm{K}$ & 2-fold & 3-fold & 4-fold & 5-fold \\
\hline 150 & $13 \%$ & $33 \%$ & $36 \%$ & $18 \%$ \\
300 & $16 \%$ & $31 \%$ & $42 \%$ & $11 \%$ \\
600 & $25 \%$ & $32 \%$ & $31 \%$ & $12 \%$ \\
\hline
\end{tabular}
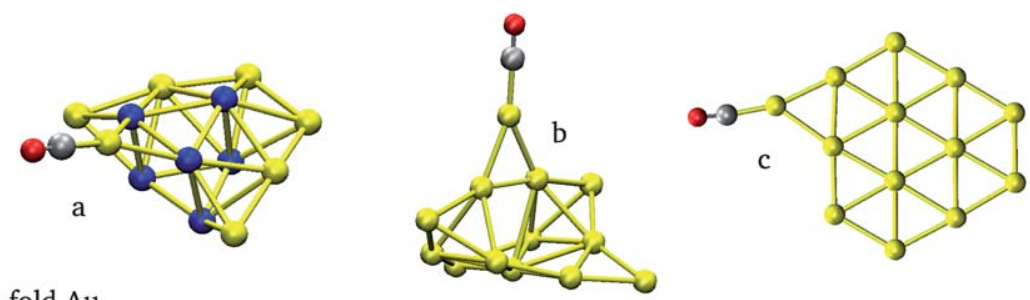

2-fold $\mathrm{Au}$
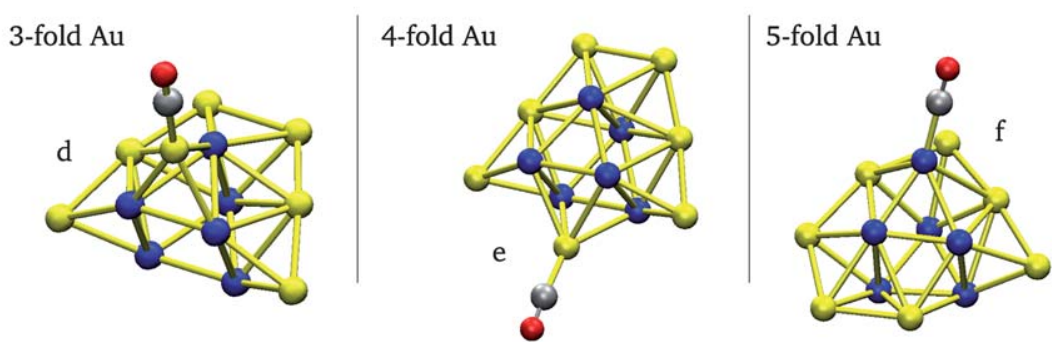

Fig. 3 Low-energy isomers for $\mathrm{Au}_{13} \mathrm{CO}$ grouped according to the coordination of the gold atom that is bonded to the carbon atom. Highlighted are the gold atoms that constitute the triangular prism.

less likely. For planar $\mathrm{Au}_{13} \mathrm{CO}$ structures like isomer $c$ in Fig. 3, it holds an argument similar to pristine clusters: Despite some of the planar structures having a total energy comparable to that of the most stable 3D structures, they are largely unfavored on entropic grounds. In Table 2 we show the energy differences among the clusters displayed in Fig. 3. In the third column of this table we report the energy difference between the $\mathrm{Au}_{13}$ remainder after removal of $\mathrm{CO}$ and the lowest energy $\mathrm{Au}_{13}$ (i.e. isomer $a$ in Fig. 1).

In practice, $\mathrm{CO}$ is removed from each isomer appearing in Fig. 3 and the remaining $\mathrm{Au}_{13}$ is geometrically optimized. In all cases, the relaxed $\mathrm{Au}_{13}$ structure maintains the same topology as in $\mathrm{Au}_{13} \mathrm{CO}$. By comparing the numbers in columns number 2 and 3 of Table 2, it is clear that a conspicuous reordering of the energy hierarchy amongst structures takes place upon adsorption of one single CO. For instance, isomer $d$ without $\mathrm{CO}$ is not one of the most stable $\mathrm{Au}_{13}$ isomers, but its stronger bonding with $\mathrm{CO}$ makes it the lowest-energy structure.

The different kinds of bonding topology are related to different bonding strengths between $\mathrm{CO}$ and the clusters. In this respect, the role of each isomer in a possible catalytic reaction (e.g. after adsorption of a oxygen molecule) is certainly different. Only by considering the population of different structures at the temperature of the reaction, one can think to model the process in an accurate (and predictive) way.

$\mathrm{Au}_{13} \mathrm{CO}$ clusters are fluxional, but they are overall more rigid than pristine $\mathrm{Au}_{13}$ clusters. In other words, they start showing fluxional behavior at higher temperatures than $\mathrm{Au}_{13}$ clusters. In particular, in the presence of certain bonding motifs, 
Table 2 The first column refers to the $\mathrm{Au}_{13} \mathrm{CO}$ isomers depicted in Fig. 3. The second coluntrieis Online the difference in total energy of $\mathrm{Au}_{13} \mathrm{CO}$ isomers from the lowest-energy one (namely isomer $d$ in Fig. 3). The third column contains the energy difference between the $\mathrm{Au}_{13}$ remainder after removal of $\mathrm{CO}$ (geometrically optimized, but still at the $\mathrm{Au}_{13} \mathrm{CO}$ geometry) and the lowestenergy $\mathrm{Au}_{13}$, i.e. isomer $a$ in Fig. 1

\begin{tabular}{lll}
\hline Isomer & $\Delta E[\mathrm{eV}]$ & $\Delta E^{*}[\mathrm{eV}]$ \\
\hline$a$ & 0.08 & 0.16 \\
$b$ & 0.11 & 0.27 \\
$c$ & 0.01 & -0.02 \\
$d$ & 0.00 & 0.19 \\
$e$ & 0.03 & 0.06 \\
$f$ & 0.07 & 0.00 \\
\hline
\end{tabular}

like those of isomers $d$ and $e$ in Fig. 3, the structure is particularly rigid (in 20 ps long MD run at $T=300 \mathrm{~K}$ these structures remain unchanged, while the corresponding pristine cluster isomers change into different structures in few ps). Clusters with other bonding motifs, like that of isomer $f$, are more fluxional, even though the temperature at which the fluxional behavior appears is significantly higher than for the corresponding $\mathrm{Au}_{13}$ structure.

\subsection{Stoichiometries at $300 \mathrm{~K}$}

When gold clusters are in contact with an atmosphere composed of $\mathrm{CO}$ and $\mathrm{O}_{2}$ gases, these ligands may adsorb onto the cluster, and the cluster may restructure completely. The preferred compositions $\mathrm{Au}_{N} \mathrm{O}_{x}(\mathrm{CO})_{y}$ adopted by the clusters in different experimental conditions are obtained using eqn (1), and the results are summarized in phase diagrams as the one shown in Fig. 4a. In the following we will focus our discussion on the case of $\mathrm{Au}_{2}$, but we will also briefly comment on trends that we find for $\mathrm{Au}, \mathrm{Au}_{3}$, and $\mathrm{Au}_{4}$. A detailed analysis of the results obtained for all cluster sizes will be published elsewhere. ${ }^{43}$
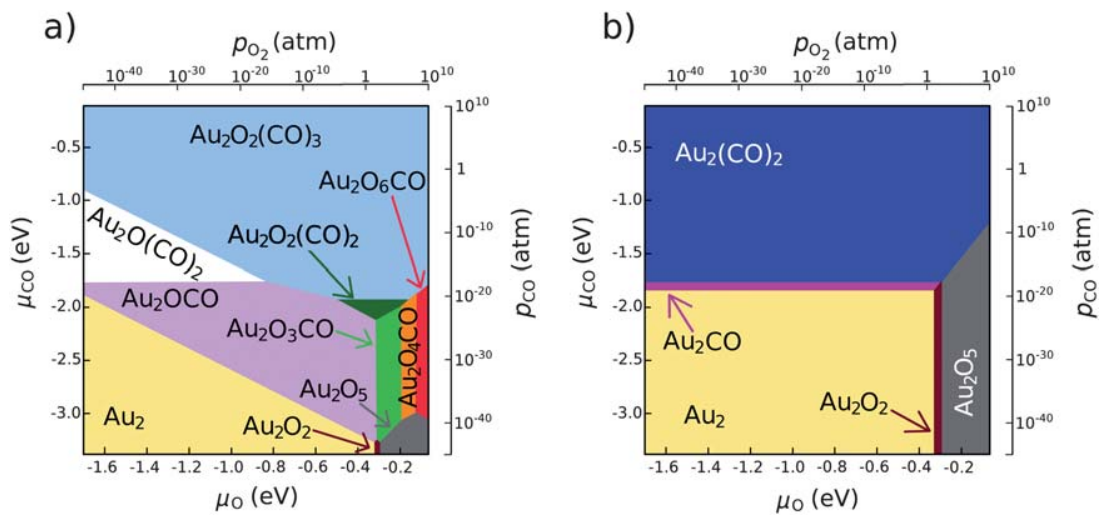

Fig. 4 Phase diagrams obtained for $\mathrm{Au}_{2} \mathrm{O}_{x}(\mathrm{CO})_{y}$ clusters at $300 \mathrm{~K}$. Each region shows the preferred composition under the partial pressures of $\mathrm{O}_{2}$ and $\mathrm{CO}$ given by the $p_{\mathrm{O}_{2}}, p_{\mathrm{CO}}$ bars. The $\mu$ scales do not correspond to absolute chemical potentials, but rather to the change in free energy at $(T, p)$ with respect to the absolute zero temperature and standard pressure: $\Delta \mu$ $(T, p)=\mu(T, p)-\mu\left(T, p^{\circ}\right)$, with $\mu\left(T, p^{\circ}\right)=E^{D F T}+E^{Z P E}$. The phase diagram has been built considering a) all isomers found in the sampling, b) only those isomers from which $\mathrm{CO}_{2}$ dissociation does not take place (see main text). 
Fig. 4a shows the phase diagram obtained for $\mathrm{Au}_{2}$ at $300 \mathrm{~K}$ and under a wide Online range of partial pressures of $\mathrm{CO}$ and $\mathrm{O}_{2}$. The phase diagram reports, for each $\left(p_{\mathrm{O}_{2}}, p_{\mathrm{CO}}\right)$ pair, the composition with the lowest free energy. This is done with a color code, such that an area with a given color identifies univocally a composition, which is indicated on (or near) the area. The approach behind Fig. 4a is also called constrained thermodynamics as it does not allow $\mathrm{O}_{2}$ and $\mathrm{CO}$ to react in the gas phase, nor does it allow $\mathrm{CO}_{2}$ to desorb (at least in Fig. 4a). The molecular structures corresponding to the different regions of Fig. $4 \mathrm{a}$ are shown in Table 3. In the region of low $p_{\mathrm{O}_{2}}$ and low $p_{\mathrm{CO}}$ the most stable structure is the pristine cluster. Adsorption of $\mathrm{O}_{2}$ and $\mathrm{CO}$ takes place when the partial pressures of the reactive gases increase.

Contrary to intuition, the first stable composition that is found by increasing $p_{\mathrm{CO}}$ at very low $p_{\mathrm{O}_{2}}$ (i.e. following upwards the left edge of the phase diagram) is not $\mathrm{Au}_{2} \mathrm{CO}$, but rather $\mathrm{Au}_{2} \mathrm{OCO}$ (violet field in Fig. 4a). This apparent oddity is due to the presence of the $\mathrm{CO}_{2}$ moiety in $\mathrm{Au}_{2} \mathrm{OCO}$ (see Table 3) whose formation energy stabilizes the cluster more than the adsorption energy of a single $\mathrm{CO}$. Consistently, at very low $p_{\mathrm{CO}}$ (bottom edge), $\mathrm{Au}_{2} \mathrm{OCO}$ becomes stable overcoming species containing only oxygen. Although $\mathrm{Au}_{2} \mathrm{OCO}$ is the most stable composition even at very low partial pressures of one of the two reactant gases, its observation in experiment may be hindered: At pressures lower than those found in intergalactic space, the collision probability of either $\mathrm{O}$ (near the left edge of the phase diagram) or $\mathrm{CO}$ (near the bottom edge) with the $\mathrm{Au}_{2}$ cluster is vanishingly small, and reaching thermodynamical equilibrium may require timescales longer than the age of the universe. However, according to thermodynamics the co-adsorption of $\mathrm{O}$ and $\mathrm{CO}$ is preferred to adsorption of $\mathrm{CO}$ alone, and $\mathrm{Au}_{2} \mathrm{CO}$ does not appear in the phase diagram.

Table 3 Preferred isomers and spin multiplicities for all the compositions appearing in Fig. 4a

\begin{tabular}{|c|c|c|}
\hline Composition & Isomer & $(2 S+1)$ \\
\hline $\mathrm{Au}_{2}$ & 0 & 1 \\
\hline $\mathrm{Au}_{2} \mathrm{O}_{2}$ & & 3 \\
\hline $\mathrm{Au}_{2} \mathrm{O}_{5}$ & & 5 \\
\hline $\mathrm{Au}_{2} \mathrm{CO}$ & 0 & 1 \\
\hline $\mathrm{Au}_{2}(\mathrm{CO})_{2}$ & & 1 \\
\hline $\mathrm{Au}_{2} \mathrm{OCO}$ & & 1 \\
\hline $\mathrm{Au}_{2} \mathrm{O}_{3} \mathrm{CO}$ & & 3 \\
\hline $\mathrm{Au}_{2} \mathrm{O}_{4} \mathrm{CO}$ & & 3 \\
\hline $\mathrm{Au}_{2} \mathrm{O}_{6} \mathrm{CO}$ & & 5 \\
\hline $\mathrm{Au}_{2} \mathrm{O}(\mathrm{CO})_{2}$ & & 1 \\
\hline $\mathrm{Au}_{2} \mathrm{O}_{2}(\mathrm{CO})_{2}$ & & 1 \\
\hline $\mathrm{Au}_{2} \mathrm{O}_{2}(\mathrm{CO})_{3}$ & & 1 \\
\hline & & 1 \\
\hline
\end{tabular}


The phase diagram in Fig. 4a shows the existence of $\mathrm{Au}_{2} \mathrm{O}_{2}$ and $\mathrm{Au}_{2} \mathrm{O}_{5}$ chlow Online ters, while $\mathrm{Au}_{2} \mathrm{O}, \mathrm{Au}_{2} \mathrm{O}_{3}$, and $\mathrm{Au}_{2} \mathrm{O}_{4}$ are absent. Thus, a gradual increase in $p_{\mathrm{O}_{2}}$ does not translate into successive adsorption of $\mathrm{O}$ atoms. The reason is that the thermodynamical stability of the $\mathrm{Au}_{2} \mathrm{O}_{x}$ structures does not increase linearly with the number of $\mathrm{O}$ atoms in the cluster. Instead, some stoichiometries are noticeably more stable than others, and only the most stable ones at a given $p_{\mathrm{O}_{2}}$, $p_{\mathrm{CO}}$ appear in the phase diagram. A similar behavior is found for $\mathrm{Au}, \mathrm{Au}_{3}$, and $\mathrm{Au}_{4}$ clusters. The presence in Fig. $4 \mathrm{a}$ of $\mathrm{Au}_{2} \mathrm{O}_{5}$ indicates that dissociative adsorption of $\mathrm{O}_{2}$ is possible for $\mathrm{Au}_{2}$ clusters at room temperature. However, it might be a slow process, due to the dissociation barrier and spin selection rules $\left(\mathrm{Au}_{2} \mathrm{O}_{5}\right.$ is a quintuplet, see Table 3). Dissociative adsorption of $\mathrm{O}_{2}$ appears in the phase diagrams of $\mathrm{Au}_{3}$ and $\mathrm{Au}_{4}$, but not in the phase diagram of the single, neutral gold atom, for which molecular adsorption of $\mathrm{O}_{2}$ is energetically more favorable than dissociative.

We also note here that the region corresponding to $\mathrm{Au}_{2} \mathrm{O}_{2}$ in Fig. 4a is defined within a range of $\mu_{O}$ of about $0.04 \mathrm{eV}$, below the accuracy in our calculations (our computational level has an uncertainty of about $\pm 0.1 \mathrm{eV}$, see Section 2) so that we cannot ensure its appearance or absence in the phase diagram.

In the $p_{\mathrm{O}_{2}}, p_{\mathrm{CO}}$ region between 0.01 and 100 atm the preferred structure $\mathrm{Au}_{2} \mathrm{O}_{2}(\mathrm{CO})_{3}$ results from co-adsorption of both $\mathrm{CO}$ and $\mathrm{O}_{2}$. The presence of co-adsorbed species in the mentioned pressure region is a feature that is also found in the phase diagrams of $\mathrm{Au}, \mathrm{Au}_{3}$, and $\mathrm{Au}_{4}$. Interestingly, in $\mathrm{Au}_{2} \mathrm{O}_{2}(\mathrm{CO})_{3}$ the $\mathrm{Au}-\mathrm{Au}$ bond is broken (see Table 3). We find that $\mathrm{Au}_{3}$ and $\mathrm{Au}_{4}$ clusters also undergo noticeable distortions due to ligand adsorption, which stresses the need of a sampling method that allows the system to go through major structural changes when searching for candidate reaction intermediates. This finding is in agreement with the work of Zhai et al. ${ }^{21}$ who arrived to a similar conclusion for one particular size of anionic gold cluster, $\mathrm{Au}_{6}^{-}$, with up to $6 \mathrm{CO}$ molecules adsorbed onto it.

\subsection{Implications for catalysis}

Many of the stoichiometries in Fig. 4a correspond to structures on which the reaction gases have formed $\mathrm{CO}_{2}$ and $\mathrm{CO}_{3}$ arrangements (see Table 3). All these clusters can be considered intermediates from which $\mathrm{CO}_{2}$, the reaction product, may desorb into the atmosphere. In this section we consider the possibility of $\mathrm{CO}_{2}$ desorption from these structures. Again, we focus our discussion on the $p_{\mathrm{O}_{2}}, p_{\mathrm{CO}}$ region in the phase diagram between 0.01 and $100 \mathrm{~atm}$, as this is the most relevant pressure region for catalysis at room temperature. In the case of $\mathrm{Au}_{2}$ the preferred structure in this region is $\mathrm{Au}_{2} \mathrm{O}_{2}(\mathrm{CO})_{3}$. It is worth noting here that the stability of $\mathrm{Au}_{2} \mathrm{O}_{2}(\mathrm{CO})_{3}$ extends for orders of magnitude outside the pressure region of interest. Therefore, our predictions will not be affected by the mentioned DFT-GGA error in the formation energies (see Section 2).

If $\mathrm{CO}_{2}$ is formed on the cluster and released into the atmosphere, eventually there will be a certain partial pressure of $\mathrm{CO}_{2}$ in equilibrium with the cluster: $\mathrm{R}-\mathrm{CO}_{2} \rightleftharpoons$ $\mathrm{R}+\mathrm{CO}_{2}$. Using the desorption energy and the partition function of $\mathrm{CO}_{2}$, we can compute $p_{\mathrm{CO}_{2}}{ }_{2}$, the partial pressure at which $\mathrm{CO}_{2}$ is in thermodynamical equilibrium with the isomer $\mathrm{R}-\mathrm{CO}_{2}$. If in the experimental setup the pressure of $\mathrm{CO}_{2}$ is below this $p_{\mathrm{CO}_{2}}{ }^{e q}, \mathrm{CO}_{2}$ dissociation will be thermodynamically favorable. In the case of $\mathrm{Au}_{2} \mathrm{O}_{2}(\mathrm{CO})_{3}$ we can write:

$$
\mathrm{Au}_{2} \mathrm{O}_{2}(\mathrm{CO})_{3} \rightleftharpoons \mathrm{Au}_{2} \mathrm{O}(\mathrm{CO})_{2}+\mathrm{CO}_{2}: p_{\mathrm{CO}_{2}}^{e q}=4.6 \times 10^{9} \mathrm{~atm} .
$$

Taking as a reference the value of $p_{\mathrm{CO}_{2}}$ estimated in the Earth's atmosphere, $p_{\mathrm{CO}_{2}} \cong 10^{-3} \mathrm{~atm}$, it follows that $\mathrm{CO}_{2}$ will desorb from $\mathrm{Au}_{2} \mathrm{O}_{2}(\mathrm{CO})_{3}$. Let us consider the remaining cluster $\mathrm{Au}_{2} \mathrm{O}(\mathrm{CO})_{2}$ : 


$$
\mathrm{Au}_{2} \mathrm{O}(\mathrm{CO})_{2} \rightleftharpoons \mathrm{Au}_{2} \mathrm{CO}+\mathrm{CO}_{2}: p_{\mathrm{CO}_{2}}^{e q}=8.9 \times 10^{11} \mathrm{~atm},
$$

from which $\mathrm{CO}_{2}$ will also readily desorb at $p_{\mathrm{CO}_{2}} \cong 10^{-3} \mathrm{~atm}$.

For all structures in Fig. 4a that contain $\mathrm{CO}_{2}$, we calculate the equilibrium partial pressure of $\mathrm{CO}_{2}$ and remove from the phase diagram all the isomers from which $\mathrm{CO}_{2}$ desorbs. In the particular case of $\mathrm{Au}_{2}$, all clusters containing the $\mathrm{CO}_{2}$ moiety are not stable with respect to $\mathrm{CO}_{2}$ dissociation at the reference $p_{\mathrm{CO}_{2}}$. With this criterion, we construct a second phase diagram, shown in Fig. 4b, in which all the isomers are stable with respect to both adsorption of $\mathrm{O}_{2}$ and $\mathrm{CO}$, and desorption of $\mathrm{CO}_{2}$ (at the reference pressure of $10^{-3} \mathrm{~atm}$ ). In this second phase diagram, $\mathrm{Au}_{2} \mathrm{CO}$, i.e. the remaining cluster after dissociation of two $\mathrm{CO}_{2}$ molecules as described above, is not the most stable structure in the pressure region of interest, and it must adsorb one $\mathrm{CO}$ molecule in order to reach the most stable composition, $\mathrm{Au}_{2}(\mathrm{CO})_{2}$.

The latter stoichiometry is to be regarded as the thermodynamically stable catalyst at the mentioned pressure conditions. Indeed, this is the most stable composition which does not contain $\mathrm{CO}_{2}$ moieties. Further adsorption of $\mathrm{CO}$ and $\mathrm{O}_{2}$ molecules will eventually lead to the $\mathrm{Au}_{2} \mathrm{O}_{2}(\mathrm{CO})_{3}$ structure (light blue in Fig. 4a) from which $\mathrm{CO}_{2}$ would desorb, and so forth.

We can identify a cycle that runs between the two extreme compositions, $\mathrm{Au}_{2}(\mathrm{CO})_{2}$ and $\mathrm{Au}_{2} \mathrm{O}_{2}(\mathrm{CO})_{3}$, by successive adsorptions of $\mathrm{O}_{2}$ and $\mathrm{CO}$ molecules and desorption of $\mathrm{CO}_{2}$ molecules. Taking into account that the total spin momentum must be conserved in each reaction step, the cycle can be written as in Fig. 5, where we indicate the energy difference (in eV) between products and reactants for each elementary step. Those species in a triplet spin state are marked with the symbol $\dagger$, while the rest of species are in a singlet state. There are two elementary step not energetically favorable, with energy differences of +0.70 and $+0.10 \mathrm{eV}$. Such energy differences can typically be overcome in the course of a catalytic process at $300 \mathrm{~K}$. The rest of reaction steps in the cycle are energetically favorable. As a consequence, the proposed mechanism is driven by an overall gain of energy. Although there is not a unique possibility for a reaction path connecting the $\mathrm{Au}_{2}(\mathrm{CO})_{2}$ and $\mathrm{Au}_{2} \mathrm{O}_{2}(\mathrm{CO})_{3}$ structures, we present the above mechanism as an example of likely reaction path according to thermodynamic considerations. Our predictions will be assessed with a complementary kinetic study of energy barriers and the consideration of alternative reaction paths.

Comparing the two phase diagrams in Fig. 4, we can distinguish two classes of $\mathrm{Au}_{2} \mathrm{O}_{x}(\mathrm{CO})_{y}$ clusters: On one hand, the intermediates, shown in Fig. 4a. The formation of these structures is very favored thermodynamically from adsorption of $\mathrm{O} / \mathrm{O}_{2}$ and $\mathrm{CO}$. They are decomposed by means of $\mathrm{CO}_{2}$ desorption, and again reformed by $\mathrm{O}_{2}$ and $\mathrm{CO}$ adsorption, i.e. for this group of intermediates the reaction would follow a Langmuir-Hirshfeld mechanism. On the other hand, the clusters in Fig. $4 \mathrm{~b}$ constitute the equilibrium structures of the catalyst. In the $p_{\mathrm{O}_{2}}, p_{\mathrm{CO}}$ region between 0.01 and $100 \mathrm{~atm}$, the catalyst is $\mathrm{Au}_{2}(\mathrm{CO})_{2}$. This class of clusters may participate in

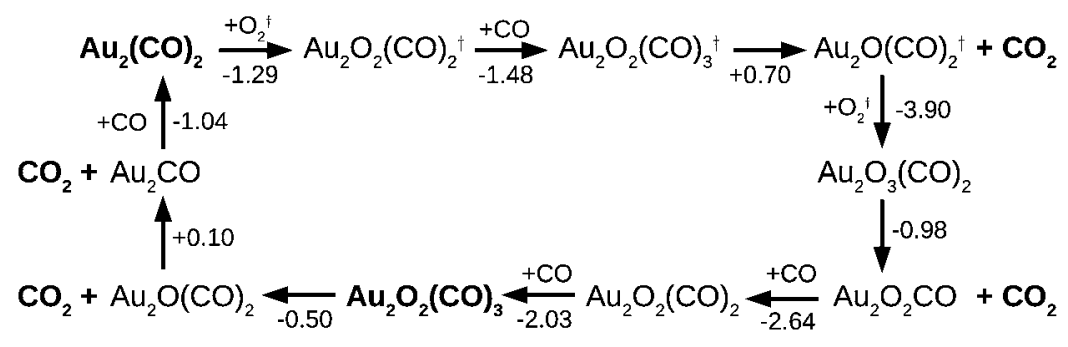

Fig. 5 Reaction cycle connecting the catalyst $\left(\mathrm{Au}_{2}(\mathrm{CO})_{2}\right)$ and the main reaction intermediate $\left(\mathrm{Au}_{2} \mathrm{O}_{2}(\mathrm{CO})_{3}\right)$ at $300 \mathrm{~K}$. 
the reaction if either additional ligands adsorb on their surface to form one ofvtiae Online intermediate structures, or if the already adsorbed ligands react with another ligand from the gas phase (Eley-Rideal mechanism).

At higher temperatures, both the intermediate and the catalyst can be different from the structures identified at room temperature (see discussion of section 3). Different reaction pathways might then dominate; the study of the influence of temperature on the identification of the relevant species for the reaction will be part of a future contribution. In addition, it must be stressed that the temperature of a small cluster in the gas phase undergoes very strong fluctuations: When a ligand is adsorbed, the energy released is rapidly distributed among the vibrational degrees of freedom (on a time scale of picoseconds) and the cluster heats up. In contrast, thermal equilibration with the buffer gas requires many collisions and a timescale of several nanoseconds. Therefore, clusters in the gas phase cannot be regarded as a system at a constant temperature.

As we have illustrated in this section, our phase diagrams do not only provide information on the relative stabilities of different cluster compositions, but they can also suggest those reaction paths that may be propitious at the environmental conditions of interest. However, these predictions are based on the assumption that during the experiment the system is able to reach thermodynamical equilibrium. This might not be the case if the catalytic reaction is too fast. Furthermore, the system might need to surmount large energy barriers in order to reach the thermodynamically preferred reaction intermediates, so that those structures might be kinetically hindered during the catalytic reaction. Our thermodynamical predictions need to be complemented with an extensive kinetic study considering reaction paths, barriers, and the conservation of spin in the processes of ligand adsorption, desorption, and reaction. This will be the subject of a future contribution from our group.

\section{Conclusions}

We have studied free pristine gold clusters and cluster+ligands complexes at finite temperature and in contact with an atmosphere composed of $\mathrm{O}_{2}$ and $\mathrm{CO}$. The preferred stoichiometries and structures are determined as a function of the environmental temperature and partial pressures of the reacting gases. Pristine as well as complexed gold clusters show a fluxional character when their finite temperature behavior is explicitly considered. Even at low temperatures $($ e.g. $100 \mathrm{~K})$ gold clusters such as $\mathrm{Au}_{13}$ and complexes such as $\mathrm{Au}_{13} \mathrm{CO}$ appear as a statistical ensemble of structures, dynamically transforming into one another, rather than as a dominant structure. On the basis of the analysis of $\mathrm{Au}_{13}$, we find a statistical argument for explaining why two-dimensional structures that have similar bonding energies to threedimensional structures are less likely to be seen in a canonical sampling.

Our statistical argument is not limited to $\mathrm{Au}_{13}$; the necessary condition to invoke it is having a fluxional system in which classes of topologically different structures have members with almost the same energy.

The adsorption energy of $\mathrm{CO}$ on $\mathrm{Au}_{N}$ is larger than that of $\mathrm{O}_{2}$. However, if both $\mathrm{O}_{2}$ and $\mathrm{CO}$ are in the surrounding gas phase the co-adsorption of both species is energetically favored. Adsorption of $\mathrm{O}_{2}$ (without $\mathrm{CO}$ ) happens only at high $\mathrm{O}_{2}$ partial pressures, and is preferred in a dissociative manner. Contrary to what might be the general chemical intuition, a progressive increase in $p_{\mathrm{O}_{2}}$ does not lead to the appearance of clusters containing a linearly increasing number of $\mathrm{O}$ atoms. Instead, certain structures are favored, due to their marked energetic stability.

When both $\mathrm{O}_{2}$ and $\mathrm{CO}$ are adsorbed, these ligands often react on the cluster surface to form adsorbed $\mathrm{CO}_{2}$ and $\mathrm{CO}_{3}$ species. Such structures are considered reaction intermediates in a mechanism of the Langmuir-Hinshelwood type. A second type of metastable structures is identified as those clusters from which $\mathrm{CO}_{2}$ desorption is not possible or not thermodynamically favorable. This last class of structures represents the equilibrium structures of the catalyst, and can either undergo ligand 
adsorption to form reaction intermediates, or react with ligands in the gas phasevidu Online an Eley-Rideal mechanism.

In addition, at $300 \mathrm{~K}$ and in the $p_{\mathrm{O}_{2}}, p_{\mathrm{CO}}$ pressure region between 0.01 and $100 \mathrm{~atm}$, we can propose a reaction path which cycles between the equilibrium catalyst and the reaction intermediate, on the basis of thermodynamical arguments. A thorough study of different reaction pathways, completing our thermodynamical information with kinetic studies, will be the subject of a future contribution.

\section{Acknowledgements}

E.C.B. thanks the Alexander von Humboldt foundation for a research fellowship. This work is supported by the Deutsche Forschungsgemeinschaft through the Cluster of Excellence UNICAT hosted by the Technical University Berlin.

\section{References}

1 H. Huber, D. McIntosh and G. A. Ozin, Inorg. Chem., 1977, 16, 975-979.

2 M. Haruta, T. Kobayashi, H. Sano and N. Yamada, Chem. Lett., 1987, 2, 405-408.

3 G. C. Bond and D. Thompson, Catal. Rev. Sci. Eng., 1999, 41, 319.

4 S. Carrettin, P. McMorn, P. Johnston, K. Griffin and G. J. Hutchings, Chem. Commun., 2002, 696.

5 A. Abad, P. Concepcion, A. Corma and H. Garcia, Angew. Chem., Int. Ed., 2005, 44, 4066.

6 M. D. Hughes, Y. J. Xu, P. Jenkins, P. McMorn, P. Landon, D. I. Enache, A. F. Carley, G. A. Attard, G. J. Hutchings, F. King, E. H. Stitt, P. Johnston, K. Griffin and C. J. Kiely, Nature, 2005, 437, 1132.

7 M. Turner, V. B. Golovko, O. P. H. Vaughan, P. Abdulkin, A. Berenguer-Murcia1, M. S. Tikhov, B. F. G. Johnson and R. M. Lambert, Nature, 2008, 454, 981.

8 L. Lian, P. A. Hackett and D. M. Rayner, J. Chem. Phys., 1993, 99, 2583-2590.

9 T. H. Lee and K. M. Ervin, J. Phys. Chem., 1994, 98, 10023-10031.

10 H. Häkkinen and U. Landman, J. Am. Chem. Soc., 2001, 123, 9704-9705.

11 W. T. Wallace and R. L. Whetten, J. Am. Chem. Soc., 2002, 124, 7499-7505.

12 G. Mills, M. S. Gordon and H. Metiu, Chem. Phys. Lett., 2002, 359, 493-499.

13 L. D. Socaciu, J. Hagen, T. M. Bernhardt, L. Wöste, U. Heiz, H. Häkkinen and U. Landman, J. Am. Chem. Soc., 2003, 125, 10437-10445.

14 H. Häkkinen, S. Abbet, A. Sanchez, U. Heiz and U. Landman, Angew. Chem., Int. Ed., 2003, 42, 1297-1300.

15 B. Yoon, H. Häkkinen and U. Landman, J. Phys. Chem. A, 2003, 107, 4066-4071.

16 R. Meyer, C. Lemire, S. K. Shaikhutdinov and H. J. Freund, Gold Bull., 2004, 37, 72-124.

17 S. Arrii, F. Morfin, A. J. Renouprez and J. L. Rousset, J. Am. Chem. Soc., 2004, 126, $1199-$ 1205.

18 T. M. Bernhardt, Int. J. Mass Spectrom., 2005, 243, 1-29.

19 M. L. Kimble, N. A. Moore, G. E. Johnson, A. W. Castleman, Jr., C. Bürgel, R. Mitric and V. Bonaĉič-Koutecký, J. Chem. Phys., 2006, 125, 204311-14.

20 S. Chrétien, S. K. Buratto and H. Metiu, Curr. Opin. Solid State Mater. Sci., 2007, 11, 6275.

21 H. J. Zhai, L. L. Pan, B. Dai, B. Kiran, J. Li and L. S. Wang, J. Phys. Chem. C, 2008, 112, $11920-11928$.

22 F. Wang, D. Zhang, X. Xu and Y. Ding, J. Phys. Chem. C, 2009, 113, 18032-18039.

23 A. Vargas, G. Santarossa, M. Iannuzzi and A. Baiker, Phys. Rev. B: Condens. Matter Mater. Phys., 2009, 80, 195421.

24 C. M. Weinart and M. Scheffler, Defects in Seminconductors, Mat. Sci. Forum 10-12, 1986, $25-30$

25 M. Scheffler and J. Dabrowski, Philos. Mag. A, 1988, 58, 107.

26 K. Reuter and M. Scheffler, Phys. Rev. B: Condens. Matter, 2001, 65, 035406.

27 K. Reuter and M. Scheffler, Phys. Rev. B: Condens. Matter, 2003, 68, 045407.

28 D. J. Wales and J. Doye, J. Phys. Chem. A, 1997, 101, 5111-5116.

29 P. Gruene, D. M. Rayner, B. Redlich, A. F. G. van Der Meer, J. T. Lyon, G. Meijer and A. Fielicke, Science, 2008, 321, 674.

30 E. Marinari and G. Parisi, Europhys. Lett., 1992, 19, 451.

31 Y. Sugita and Y. Okamoto, Chem. Phys. Lett., 1999, 314, 141.

32 V. Blum, R. Gehrke, F. Hanke, P. Havu, V. Havu, X. Ren, K. Reuter and M. Scheffler, Comput. Phys. Commun., 2009, 180, 2175-2196. 
33 J. P. Perdew, K. Burke and M. Ernzerhof, Phys. Rev. Lett., 1996, 77, 3865-3868. View Online 34 J. P. Perdew, K. Burke and M. Ernzerhof, Phys. Rev. Lett., 1997, 78, 1396-1396.

35 E. van Lenthe, E. J. Baerends and J. G. Snijders, J. Chem. Phys., 1993, 99, 4597-4610.

36 R. M. Olson, S. Varganov, M. S. Gordon, H. Metiu, S. Chrétien, P. Piecuch, K. Kowalski, S. A. Kucharski and M. Musial, J. Am. Chem. Soc., 2005, 127, 1049-1052.

37 F. Mendizabal, Organometallics, 2001, 20, 261-265.

38 A. Tkatchenko and M. Scheffler, Phys. Rev. Lett., 2009, 102, 073005.

39 R. Fowler and E. A. Guggenheim, Statistical thermodynamics, Cambridge University Press, Cambridge, 1949.

40 J. Rogal and K. Reuter, Experiment, Modeling and Simulation of Gas-Surface Interactions for Reactive Flows in Hypersonic Flights, Educational notes RTO-EN-AVT-142, Neuillisur-Seine, France, 2007, pp. 2-1-18.

41 G. Bussi, D. Donadio and M. Parrinello, J. Chem. Phys., 2007, 126, 014101.

42 D. J. Sindhikara, D. J. Emerson and A. Roitberg, J. Chem. Theory Comput., 2010, 6, 2804.

43 E. C. Beret, L. M. Ghiringhelli and M. Scheffler, in preparation.

44 L. M. Ghiringhelli, P. Gruene, J. T. Lyon, G. Meijer, A. Fielicke and M. Scheffler, in preparation.

45 D. Häberlen, S. C. Chung, M. Stener and N. Rösch, J. Chem. Phys., 1997, 106, 5189.

46 J. Oviedo and R. E. Palmer, J. Chem. Phys., 2002, 117, 9548.

47 M. Chang and M. Y. Chou, Phys. Rev. Lett., 2004, 93, 133401.

48 M. Fernandez, J. M. Soler, I. L. Garzon and L. C. Balbas, Phys. Rev. B: Condens. Matter Mater. Phys., 2004, 70, 165403.

49 L. Xiao, B. Tollberg, X. K. Hu and L. C. Wang, J. Chem. Phys., 2006, 124, 114309.

50 M. Gruber, G. Heimel, L. Romaner, J. Brédas and E. Zojer, Phys. Rev. B: Condens. Matter Mater. Phys., 2008, 77, 165411.

51 S. Gilb, P. Weis, F. Furche, R. Ahlrichs and M. M. Kappes, J. Chem. Phys., 2002, 116, 4094.

52 M. P. Johansson, A. Lechtken, D. Schooss, M. M. Kappes and F. Furche, Phys. Rev. A: At., Mol., Opt. Phys., 2008, 77, 053202.

53 P. Koskinen, H. Häkkinen, B. Huber, B. von Issendorff and M. Moseler, Phys. Rev. Lett., 2007, 98, 015701.

54 A. Laio and M. Parrinello, Proc. Natl. Acad. Sci. U. S. A., 2002, 99, 12562. 